\title{
Sopra i Gruppi astratti di grado 32
}

\author{
(Di G. Bagnera, a Palermo.)
}

Io adopero il simbolo

$$
[\alpha, \beta, \ldots, \varepsilon]
$$

per denotare una classe $\Gamma$ di enti, ciascuno dei quali è perfettamente individuato quando sono dati $\mathrm{i}$ numeri interi $\alpha, \beta, \ldots, \varepsilon$. Sia $\Theta$ la definizione di eguaglianza di due elementi di $\Gamma$ scelta in modo che, essendo $A, B, C$ tre elementi della detta classe, siano verificate le condizioni :

1. ${ }^{\mathrm{a}}$ qualunque sia l'elemento $A$ è sempre $A=A$,

2. ${ }^{\text {a }}$ tutte le volte che è $A=B$ è anche $B=A$,

$3 .^{\mathrm{a}}$ se è $A=B$ e $B=C$ deve essere $A=C$.

Finalmente jo suppongo data un'operazione $\Omega$, perfettamente definita in $\Gamma$, tale che

$$
A \Omega B=A B
$$

sia un elemento di $\Gamma$ tutte le volte che $A$ e $B$ sono elementi di $\Gamma$. L'operazione $\Omega$ non è indipendente da $\Theta$, perchè io ammetto che si verifichino le condizioni seguenti :

$1 .^{\mathrm{a}}$ se è $A=B$ è anche $A C=B C$ e $C A=C B$,

$2 .^{\mathrm{a}}$ se è $A C=B C$ oppure $C A=C B$ è $A=B$,

$3 .^{a}$ in ogni caso è $(A B) C=A(B C)$.

Se la classe $\Gamma$ contiene soltanto un numero finito $n$ di elementi distinti rispetto a $\Theta$, essi costituiscono rispetto all'operazione $\Omega$ un Gruppo finito di grado $n$ : esiste allora un elemento $E$, ben determinato rispetto a $\Theta$, tale che

$$
A E=E A=A \text {, }
$$

qualunque sia l'elemento $A$ di $\Gamma$. Io suppongo che sia:

$$
E=[0,0, \ldots, 0] \text {. }
$$


L'ordine di un elemento $A$ è il minimo numero intero e positivo $m$ tale che $A^{m}=E$, e l'elemento inverso di $A$ è quell'elemento $A^{-1}$ tale che $A A^{-1}=E$.

Gli elementi di un qualsivoglia gruppo, il cui grado è una potenza $p^{h}$ di un numero primo $p$, si possono rappresentare con simboli

$$
[\alpha, \beta, \ldots, \varepsilon]
$$

contenenti $h$ indici $\alpha, \beta, \ldots, \varepsilon$; ma questi $h$ indici non sono sempre necessari : per es., volendo definire il gruppo ciclico di grado $32=2^{5}$, in posso scrivere le formole :

$\Theta \equiv[\alpha, \beta, \gamma, \delta, \varepsilon]=[\alpha+2 \lambda, \beta+2 \mu-\lambda, \gamma+2 \nu-\mu, \dot{\partial}+2 \beta-\nu, \varepsilon+2 \sigma-\rho]$,

$\Omega \equiv[\alpha, \beta, \gamma, \delta, \varepsilon]\left[\alpha^{\prime}, \beta^{\prime}, \gamma^{\prime}, \delta^{\prime}, \varepsilon^{\prime}\right]=\left[\alpha+\alpha^{\prime}, \beta+\beta^{\prime}, \gamma+\gamma^{\prime}, \delta^{\prime}+\delta^{\prime}, \varepsilon+\varepsilon^{\prime}\right]$,

ma sono più comode le seguenti :

$$
\begin{aligned}
& \Theta \equiv[\alpha]=[\alpha+32 \lambda], \\
& \Omega \equiv[\alpha]\left[\alpha^{\prime}\right]=[\alpha+\alpha] .
\end{aligned}
$$

Bisogna però osservare che non è sempre conveniente di ridurre al minimo il numero dei detti indici, perchè una tale riduzione va spesso a discapito della semplicità di $\theta$ ed $\Omega$.

Per il problema che consiste nel ricercare tutti i possibili gruppi $\Gamma \mathrm{di}$ grado $p^{h}$ è interessante la considerazione del gruppo $K$ intersezione di tutti $i$ divisori d'indice $p$ contenuti in un tale gruppo $\Gamma$ : ciò risulta dal fatto che il gruppo $K$ si può anche definire nella seguente maniera:

Siano $A$ e $B$ due arbitrari elementi di [' e si ponga

$$
B^{-1} A B=A A^{\prime} \text { : }
$$

il gruppo $K$ è allora il gruppo di grado minimo che contiene tutti gli elementi tali che $A^{\prime}$ ed $A^{p}$.

Io ho risoluto l'anzidetto problema per il caso di $h=5$ e $p$ qualunque

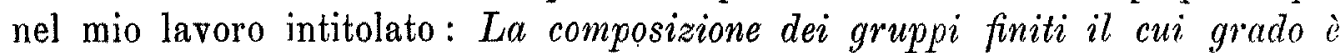
la quinta potenza di un numero primo $\left({ }^{*}\right)$; ma io ritorno ora sul caso particolare di $p=2$, per correggere due inesattezze, che, soltanto in questo caso, vi si incontrano e che sono del resto facilmente rilevabili dal lettore; inoltre, profitto della circostanza di dovere ritornare sull'argomento per dare la dimostrazione della effettiva esistenza di ciascuno dei gruppi di grado 32 scritti

(*) Questi Aninali, s. III, t. I (1898). 
nel citato lavoro, esistenza che è ivi semplicemente asserita, e per mettere maggiormente in rilievo l'intima costituzione dei detti gruppi. Un esempio chiarirà meglio il mio pensiero.

Si scrivano le formole:

$$
\begin{aligned}
& \theta \equiv[\alpha, \beta]=[\alpha+2 \lambda, \beta+16 \mu], \\
& \Omega \equiv[\alpha, \beta]\left[\alpha^{\prime}, \beta^{\prime}\right]=\left[\alpha+\alpha^{\prime},(-1)^{\alpha^{\prime}} \beta+\beta^{\prime}\right] .
\end{aligned}
$$

dove $\lambda$ e $\mu$ sono numeri interi arbitrari; allora gli elementi $[\alpha, \beta]$ che sono distinti rispetto a $\Theta$ sono soltanto 32 : essi si ottengono attribuendo per es. ad $\alpha$ i valori 0,1 ed a $\beta$ i valori $0,1,2, \ldots, 15$ indipendentemente.

$\grave{E}$ facile verificare che $\Theta$ ed $\Omega$ soddisfano alle condizioni enunciate al principio della presente Nota e quindi io ho definito un gruppo di grado 32 dove l'elemento identico è il simbolo $[0,0]$.

Volendo trovare gli elementi di secondo ordine contenuti in questo gruppo si dirà: se $[\alpha, \beta]$ è un tale elemento si ha:

$$
[0,0]=[\alpha, \beta]^{2}=\left[2 \alpha,(-1)^{\alpha} \beta+\beta\right] \text {, }
$$

e quindi, in virtù di $\Theta$, è :

$$
(-1)^{\alpha} \beta+\beta=16 \mu \text {. }
$$

Dunque, se a è pari, bisogna scegliere per $\beta$ un multiplo di 8 , ma se $\alpha$ è impari $\beta$ è arbitrario. Si hanno cos̀ 18 elementi che verificano la (1), tra $i$ quali è certamente compreso l'elemento $[0,0]$ che non è di secondo ordine; perciò nel gruppo considerato si trovano 17 elementi di secondo ordine dei quali uno e $[0,8]$ e gli altri sono rappresentati dal simbolo $[1, \beta]$ per $\mathrm{i}$ diversi valori di $\beta$.

Io mi propongo ancora la ricerca di tutti gli elementi del detto gruppo che godono la proprietà di essere permutabili con qualsiasi elemento del gruppo stesso. Se $[\alpha, \beta]$ è un tale elemento, deve essere in virtù di $\Omega$ :

$$
\left[\alpha+\alpha^{\prime},(-1)^{\alpha^{\prime} \beta}+\beta^{\prime}\right]=\left[\alpha^{\prime}+\alpha,(-1)^{\alpha} \beta^{\prime}+\beta\right]
$$

qualunque siano $\alpha^{\prime}, \beta^{\prime}$ e quindi bisogna che sia:

$$
(-1)^{\alpha} \beta^{\prime}+\beta=(-1)^{\alpha} \beta+\beta^{\prime}+16 \mu \text {. }
$$

Supponendo $\alpha^{\prime}=0$ e $\beta^{\prime}=1$ si vede che $\alpha$ deve essere un numero pari, poi facendo $\alpha^{\prime}=1$ e $\beta^{\prime}=0$ si vede che $\beta$ deve essere un multiplo di 8 ; quindi esistono due soli elementi che godono la detta proprieta $i$ quali sono:

$$
[0,0], \quad[0,8] \text {. }
$$


Se si pone:

$$
[1,0]=A, \quad[0,1]=B, \quad[0,0]=1,
$$

si trova:

$$
A^{-1} B A=B^{-1}, \quad A^{z}=1, \quad B^{16}=1,
$$

e perciò il gruppo dell'attuale esempio, che porta il numero XLII nella tabella che segue, è il gruppo diedro di grado 32 .

Il relativo gruppo $K$ si può calcolare servendosi della seconda definizione che ho dato per il detto gruppo. La conoscenza di $K$ dà subito il numero dei divisori di grado 16 contenuti nel gruppo di grado 32 : questo numero è espresso da $2^{k}-1$ dove $k$ è l'indice di $K$ rispetto al gruppo principale. Nell'esempio precedente si trova che $K$ è il gruppo ciclico di grado 8 generato dall'elemento $[0,2]$ e quindi il gruppo principale possiede tre divisori di grado 16, uno dei quali è il gruppo ciclico generato dall'elemento $[0,1]$.

Nella tabella che segue jo ho scritto accanto al numero ordinativo di ogni gruppo quanti sono, separatamente, gli elementi di $2 .^{\circ}, 4 .^{\circ}, 8 .^{\circ}$ e $16^{\circ}$ ordine in esso gruppo contenuti ed anche gl'invarianti del corrispondente gruppo $K$ che, per i gruppi di grado $p^{5}$, è sempre Abeliano. Così accanto al numero XLII si trova scritto: $(17,2,4,8)(8)$, il che significa che il corrispondente gruppo di grado 32 ha $17,2,4,8$ elementi degli ordini rispettivi $2,4,8,16$ e che il relativo gruppo $K$ possiede un solo invariante eguale ad 8.

Dopo ciò, pochi sono i gruppi della detta tabella per i quali il lettore possa concepire il sospetto che essi siano isomorf, ma questo sospetto si toglie facilmente dietro alcune considerazioni d'indole particolare, che ho esposto, dopo la tabella, in apposite osservazioni. Finalmente, la risposta all'obbiezione, se nessun gruppo di grado 32 esiste che non sia isomorfo ad uno di quelli che ora scriverò, è negativa e si trova nei ragionamenti svolti nel mio citato lavoro.

I sette Gruppi Abeliani di grado 32.

I. $(1,2,4,8)(16)$.

$$
[\alpha]=[\alpha+32 \lambda], \quad[\alpha]\left[\alpha^{\prime}\right]=\left[\alpha+\alpha^{\prime}\right] \text {. }
$$

II. $(3,4,8,16)(8)$.

$$
[\alpha, \beta]=[\alpha+16 \lambda, \beta+2 \mu], \quad[\alpha, \beta]\left[\alpha^{\prime}, \beta^{\prime}\right]=\left[\alpha+\alpha^{\prime}, \beta+\beta^{\prime}\right] \text {. }
$$


III. $(3,12,16,0)(4,2)$.

$[\alpha, \beta]=[\alpha+8 \lambda, \beta+4 \mu],[\alpha, \beta]\left[\alpha^{\prime}, \beta^{\prime}\right]=\left[\alpha+\alpha^{\prime}, \beta+\beta^{\prime}\right]$.

IV. $(7,8,16,0)(4)$.

$[\alpha, \beta, \gamma]=[\alpha+8 \lambda, \beta+2 \mu, \gamma+2 \nu]$,

$[\alpha, \beta, \gamma]\left[\alpha^{\prime}, \beta^{\prime}, \gamma^{\prime}\right]=\left[\alpha+\alpha^{\prime}, \beta+\beta^{\prime}, \gamma+\gamma^{\prime}\right]$.

V. $(7,24,0,0)(2,2)$.

$[\alpha, \beta, \gamma]=[\alpha+4 \lambda, \beta+4 \mu, \gamma+2 \nu]$,

$[\alpha, \beta, \gamma]\left[\alpha^{\prime}, \beta^{\prime}, \gamma^{\prime}\right]=\left[\alpha+\alpha^{\prime}, \beta+\beta^{\prime}, \gamma+\gamma^{\prime}\right]$.

VI. $(15,16,0,0)(2)$.

$[\alpha, \beta, \gamma, \partial]=[\alpha+4 \lambda, \beta+2 \mu, \gamma+2 \nu, \grave{o}+2 \rho]$,

$[\alpha, \beta, \gamma, \delta]\left[\alpha^{\prime}, \beta^{\prime}, \gamma^{\prime}, \delta^{\prime}\right]=\left[\alpha+\alpha^{\prime}, \beta+\beta^{\prime}, \gamma+\gamma^{\prime}, \delta+\delta^{\prime}\right]$.

VII. $(31,0,0,0)(1)$.

$[\alpha, \beta, \gamma, \delta, \varepsilon]=[\alpha+2 \lambda, \beta+2 \mu, \gamma+2 \nu, \delta+2 \rho, \varepsilon+2 \sigma]$,

$[\alpha, \beta, \gamma, \delta, \varepsilon]\left[\alpha^{\prime}, \beta^{\prime}, \gamma^{\prime}, \partial^{\prime}, \varepsilon^{\prime}\right]=\left[\alpha+\alpha^{\prime}, \beta+\beta^{\prime}, \gamma+\gamma^{\prime}, \delta+\delta^{\prime}, \varepsilon+\varepsilon^{\prime}\right]$.

I quindici Gruppi di grado 32, ciascuno dei quali contiene soltanto 8 elementi PERMUTABILI CON TUTTI GLI ELEMENTI DEL GRUPPO.

VIII. $(3,4,8,16)(8)$

$[\alpha, \beta, \gamma]=[\alpha+2 \lambda, \beta+2 \mu, \gamma+8 \nu-\mu]$

$[\alpha, \beta, \gamma]\left[\alpha^{\prime}, \beta^{\prime}, \gamma^{\prime}\right]=\left[\alpha+\alpha^{\prime}, \beta+\beta^{\prime}, \gamma+\gamma^{\prime}+4 \beta \alpha^{\prime}\right]$.

IX. $(3,12,16,0)(4,2)$.

$[\alpha, \beta, \gamma]=[\alpha+4 \lambda, \beta+2 \mu, \gamma+4 \nu-\mu]$,

$[\alpha, \beta, \gamma]\left[\alpha^{\prime}, \beta^{\prime}, \gamma^{\prime}\right]=\left[\alpha+\alpha^{\prime}, \beta+\beta^{\prime}, \gamma+\gamma^{\prime}+2 \beta \alpha^{\prime}\right]$.

$\mathrm{X} .(3,12,16,0)(4,2)$.

$[\alpha, \beta, \gamma]=[\alpha+2 \lambda, \beta+8 \mu, \gamma+2 \nu-\lambda]$,

$[\alpha, \beta, \gamma]\left[\alpha^{\prime}, \beta^{\prime}, \gamma^{\prime}\right]=\left[\alpha+\alpha^{\prime}, \beta+\beta^{\prime}, \gamma+\gamma^{\prime}+\hat{\beta} \alpha^{\prime}\right]$. 
XI. $(7,8,16,0)(4,2)$.

$[\alpha, \beta, \gamma]=[\alpha+2 \lambda, \beta+8 \mu, \gamma+2 \nu]$,

$[\alpha, \beta, \gamma]\left[\alpha^{\prime}, \beta^{\prime}, \gamma^{\prime}\right]=\left[\alpha+\alpha^{\prime}, \beta+\beta^{\prime}, \gamma+\gamma^{\prime}+\beta \alpha^{\prime}\right]$.

XII. $(7,24,0,0)(2,2,2)$.

$[x, \beta, \gamma]=[\alpha+4 \lambda, \beta+4 \mu, \gamma+2 \nu]$,

$[\alpha, \beta, \gamma]\left[\alpha^{\prime}, \beta^{\prime}, \gamma^{\prime}\right]=\left[\alpha+\alpha^{\prime}, \beta+\beta^{\prime}, \gamma+\gamma^{\prime}+\beta \alpha^{\prime}\right]$.

XIII. $(7,8,16,0)(4)$.

$[\alpha, \beta, \gamma]=[\alpha+2 \lambda, \beta+2 \mu, \gamma+8 \nu]$,

$[\alpha, \beta, \gamma]\left[\alpha^{\prime}, \beta^{\prime}, \gamma^{\prime}\right]=\left[\alpha+\alpha^{\prime}, \beta+\beta^{\prime}, \gamma+\gamma^{\prime}+4 \beta \alpha^{\prime}\right]$.

XIV. $(7,24,0,0)(2,2)$.

$[\alpha, \beta, \gamma]=[\alpha+2 \lambda, \beta+4 \mu, \gamma+4 \nu]$,

$[\alpha, \beta, \gamma]\left[\alpha^{\prime}, \beta^{\prime}, \gamma^{\prime}\right]=\left[\alpha+\alpha^{\prime}, \beta+\beta^{\prime}, \gamma+\gamma^{\prime}+2 \beta \alpha^{\prime}\right]$.

$\mathrm{XV} .(11,12,8,0)(4)$.

$[\alpha, \beta, \gamma, \delta]=[\alpha+2 \lambda, \beta+2 \mu, \gamma+4 \nu-\mu, \delta+2 \rho]$,

$\left.\left[\alpha, \beta, \gamma, \delta^{\prime}\right]\left[\alpha^{\prime}, \beta^{\prime}, \gamma^{\prime}, \delta^{\prime}\right]=\mid \alpha+\alpha^{\prime}, \beta+\beta^{\prime}, \gamma+\gamma^{\prime}+\beta \alpha^{\prime}, \delta^{\prime}+\delta^{\prime}\right]$.

XVI. $(11,20,0,0)(2,2)$.

$[\alpha, \beta, \gamma, \delta]=[\alpha+2 \lambda, \beta+2 \mu, \gamma+2 \nu, \delta+4 \rho]$,

$\left[\alpha, \beta, \gamma, \delta^{\prime}\right]\left[\alpha^{\prime}, \beta^{\prime}, \gamma^{\prime}, \delta^{\prime}\right]=\left[\alpha+\alpha^{\prime}, \beta+\beta^{\prime}, \gamma+\gamma^{\prime}+\beta \alpha^{\prime}, \delta^{\prime}+\nu^{\prime}\right]$.

XVII. $(3,28,0,0)(2,2)$.

$[\alpha, \beta, \gamma, \delta]=[\alpha+2 \lambda, \beta+2 \mu, \gamma+2 \nu-\lambda-\mu, \delta+4 \rho]$,

$[\alpha, \beta, \gamma, \delta]\left[\alpha^{\prime}, \beta^{\prime}, \gamma^{\prime}, \delta^{\prime}\right]=\left[\alpha+\alpha^{\prime}, \beta+\beta^{\prime}, \gamma+\gamma^{\prime}+\beta \alpha^{\prime}, \delta^{\prime}+\delta^{\prime}\right]$.

XVIII. $(\overline{7}, 24,0,0)(2,2)$.

$[\alpha, \beta, \gamma, \hat{0}]=[\alpha+2 \lambda, \beta+4 \mu, \gamma+2 \nu-\lambda, \delta+2 \rho]$,

$\left[\alpha, \beta, \gamma, \delta^{\prime}\right]\left[\alpha^{\prime}, \beta^{\prime}, \gamma^{\prime}, \delta^{\prime}\right]=\left[\alpha+\alpha^{\prime}, \beta+\beta^{\prime}, \gamma+\gamma^{\prime}+\beta \alpha^{\prime}, \partial^{\prime}+\delta^{\prime}\right]$.

XIX. $(15,16,0,0)(2,2)$.

$[\alpha, \beta, \gamma, \delta]=[\alpha+2 \lambda, \beta+4 \mu, \gamma+2 \nu, \delta+2 \rho]$,

$[\alpha, \beta, \gamma, \delta]\left[\alpha^{\prime}, \beta^{\prime}, \gamma^{\prime}, \delta^{\prime}\right]=\left[\alpha+\alpha^{\prime}, \beta+\beta^{\prime}, \gamma+\gamma^{\prime}+\beta \alpha^{\prime}, \delta+\delta^{\prime}\right]$. 
XX. $(15,16,0,0)(2)$.

$[\alpha, \beta, \gamma, \delta]=[\alpha+2 \lambda, \beta+2 \mu, \gamma+4 \nu, \delta+2 \rho]$,

$[\alpha, \beta, \gamma, \delta]\left[\alpha^{\prime}, \beta^{\prime}, \gamma^{\prime}, \partial^{\prime}\right]=\left[\alpha+\alpha^{\prime}, \beta+\beta^{\prime}, \gamma+\gamma^{\prime}+2 \beta \alpha^{\prime}, \partial+\partial^{\prime}\right]$.

XXI. $(23,8,0,0)(2)$.

$[\alpha, \beta, \gamma, \delta, \varepsilon]=[\alpha+2 \lambda, \beta+2 \mu, \gamma+2 \nu, \delta+2 \rho, \varepsilon+2 \sigma]$,

$\left[\alpha, \beta, \gamma, \delta^{\prime}, \varepsilon\right]\left[\alpha^{\prime}, \beta^{\prime}, \gamma^{\prime}, \delta^{\prime}, \varepsilon^{\prime}\right]=\left[\alpha+\alpha^{\prime}, \beta+\beta^{\prime}, \gamma+\gamma^{\prime}+\beta \alpha^{\prime}, \delta^{\prime}+\delta^{\prime}, \varepsilon+\varepsilon^{\prime}\right]$.

XXII. $(7,24,0,0)(2)$.

$\mid \alpha, \beta, \gamma, \delta, \varepsilon]=\mid \alpha+2 \lambda, \beta+2 \mu, \gamma+2 \nu-\lambda-\mu, \delta+2 \rho, \varepsilon+2 \sigma]$,

$\left[\alpha, \beta, \gamma, \delta^{\prime}, \varepsilon\right]\left[\alpha^{\prime}, \beta^{\prime}, \gamma^{\prime}, \delta^{\prime}, \varepsilon^{\prime}\right]=\left[\alpha+\alpha^{\prime}, \beta+\beta^{\prime}, \gamma+\gamma^{\prime}+\beta \alpha^{\prime}, \delta^{\prime}+\delta^{\prime}, \varepsilon+\varepsilon^{\prime}\right]$.

I diciannove Gruppi di grado 32, ciasouno dei quali contiene soltanto 4 elementi PERMUTABILI CON TETTI GLI ELEMENTI DEL GRUPPO.

XXIII. $(15,16,0,0)(2,2)$.

$[\alpha, \beta, \gamma, \delta, \varepsilon]=[\alpha+2 \lambda, \beta+2 \mu, \gamma+2 \nu, \delta+2 \rho, \varepsilon+2 \sigma-\nu]$, $[\alpha, \beta, \gamma, \partial, \varepsilon]\left[\alpha^{\prime}, \beta^{\prime}, \gamma^{\prime}, \partial^{\prime}, \varepsilon^{\prime}\right]=\left[\alpha+\alpha^{\prime}, \beta+\beta^{\prime}, \gamma+\gamma^{\prime}, \delta+\delta^{\prime}+\beta \alpha^{\prime}, \varepsilon+\varepsilon^{\prime}+\gamma \alpha^{\prime}\right]$.

XXIV. $(7,24,0,0)(2,2)$.

$[\alpha, \beta, \gamma, \delta, \varepsilon]=[\alpha+2 \lambda, \beta+2 \mu, \gamma+2 \nu, \delta+2 \rho, \varepsilon+2 \sigma-\lambda-\nu]$, $\left[\alpha, \beta, \gamma, \partial^{\prime}, \varepsilon\right]\left[\alpha^{\prime}, \beta^{\prime}, \gamma^{\prime}, \delta^{\prime}, \varepsilon^{\prime}\right]=\left[\alpha+\alpha^{\prime}, \beta+\beta^{\prime}, \gamma+\gamma^{\prime}, \partial+\delta^{\prime}+\beta \alpha^{\prime}, \varepsilon+\varepsilon^{\prime}+\gamma \alpha^{\prime}\right]$.

XXV. $(11,20,0,0)(2,2)$.

$[\alpha, \beta, \gamma, \delta, \varepsilon]=[\alpha+2 \lambda, \beta+2 \mu, \gamma+2 \nu, \dot{\delta}+2 \rho-\nu, \varepsilon+2 \sigma]$, $\left[\alpha, \beta, \gamma, \delta^{\prime}, \varepsilon\right]\left[\alpha^{\prime}, \beta^{\prime}, \gamma^{\prime}, \delta^{\prime}, \varepsilon^{\prime}\right]=\left[\alpha+\alpha^{\prime}, \beta+\beta^{\prime}, \gamma+\gamma^{\prime}, \delta^{\prime}+\delta^{\prime}+\beta \alpha^{\prime}, \varepsilon+\varepsilon^{\prime}+\gamma \alpha^{\prime}\right]$.

XXVI. $(11,20,0,0)(2,2)$.

$[\alpha, \beta, \gamma, \delta, \varepsilon]=[\sigma+2 \lambda, \beta+2 \mu, \gamma+2 \nu, \delta+2 p-\nu, \varepsilon+2 \sigma-\mu]$, $[\alpha, \beta, \gamma, \delta, \varepsilon]\left[\alpha^{\prime}, \beta^{\prime}, \gamma^{\prime}, \delta^{\prime}, \varepsilon^{\prime}\right]=\left[\alpha+\alpha^{\prime}, \beta+\beta^{\prime}, \gamma+\gamma^{\prime}, \delta+\partial^{\prime}+\beta \alpha^{\prime}, \varepsilon+\varepsilon^{\prime}+\gamma \alpha^{\prime}\right]$.

XXVII. $(3,28,0,0)(2,2)$

$[\alpha, \beta, \gamma, \delta, \varepsilon]=[\alpha+2 \lambda, \beta+2 \mu, \gamma+2 \nu, \delta+2 \beta-\nu, \varepsilon+2 \sigma-\lambda-\mu]$, $\left.\mid \alpha, \beta, \gamma, \delta^{\prime}, \varepsilon\right]\left[\alpha^{\prime}, \beta^{\prime}, \gamma^{\prime}, \delta^{\prime}, \varepsilon^{\prime}\right]=\left[\alpha+\alpha^{\prime}, \beta+\beta^{\prime}, \gamma+\gamma^{\prime}, \delta+\delta^{\prime}+\beta \alpha^{\prime}, \varepsilon+\varepsilon^{\prime}+\gamma \alpha^{\prime}\right]$. 
XXVIIJ. $(7,24,0,0)(2,2)$.

$[\alpha, \beta, \gamma, \delta, \varepsilon]=[\alpha+2 \lambda, \beta+2 \mu, \gamma+2 \nu, \delta+2 \rho-\nu, \varepsilon+2 \sigma-\mu-\nu]$, $[\alpha, \beta, \gamma, \delta, \varepsilon]\left[\alpha^{\prime}, \beta^{\prime}, \gamma^{\prime}, \delta^{\prime}, \varepsilon^{\prime}\right]=\left[\alpha+\alpha^{\prime}, \beta+\beta^{\prime}, \gamma+\gamma^{\prime}, \delta+\delta^{\prime}+\beta \alpha^{\prime}, \varepsilon+\varepsilon^{\prime}+\gamma \alpha^{\prime}\right]$.

XXIX. $(19,12,0,0)(2,2)$.

$[\alpha, \beta, \gamma, \delta, \varepsilon]=[\alpha+2 \lambda, \beta+2 \mu, \gamma+2 \nu, \delta+2 \beta-\mu, \varepsilon+2 \sigma-\nu]$, $\left.\left[\alpha, \beta, \gamma, \delta^{\prime}, \varepsilon\right]\left[\alpha^{\prime}, \beta^{\prime}, \gamma^{\prime}, \delta^{\prime}, \varepsilon^{\prime}\right]=\mid \alpha+\alpha^{\prime}, \beta+\beta^{\prime}, \gamma+\gamma^{\prime}, \delta+\delta^{\prime}+\beta \alpha^{\prime}, \varepsilon+\varepsilon^{\prime}+\gamma \alpha^{\prime}\right]$.

XXX. $(3,28,0,0)(2,2)$.

$[\alpha, \beta, \gamma, \partial, \varepsilon]=[\alpha+2 \lambda, \beta+2 \mu, \gamma+2 \nu, \delta+2 \rho-\mu, \varepsilon+2 \sigma-\lambda-\nu]$, $[\alpha, \beta, \gamma, \delta, \varepsilon]\left[\alpha^{\prime}, \beta^{\prime}, \gamma^{\prime}, \partial^{\prime}, \varepsilon^{\prime}\right]=\left[\alpha+\alpha^{\prime}, \beta+\beta^{\prime}, \gamma+\gamma^{\prime}, \delta+\delta^{\prime}+\beta \alpha^{\prime}, \varepsilon+\varepsilon^{\prime}+\gamma \alpha^{\prime}\right]$.

XXXI. $(19,12,0,0)(2,2)$.

$[\alpha, \beta, \gamma, \delta, \varepsilon]=[\alpha+2 \lambda, \beta+2 \mu, \gamma+2 \nu, \delta+2 \rho, \varepsilon+2 \sigma]$, $[\alpha, \beta, \gamma, \delta, \varepsilon]\left\lfloor\alpha^{\prime}, \beta^{\prime}, \gamma^{\prime}, \delta^{\prime}, \varepsilon^{\prime}\right]=\left[\alpha+\alpha^{\prime}, \beta+\beta^{\prime}, \gamma+\gamma^{\prime}, \delta+\delta^{\prime}+\beta \alpha^{\prime}, \varepsilon+\varepsilon^{\prime}+\gamma \alpha^{\prime}\right]$.

XXXII. $(11,12,8,0)(4)$.

$[\alpha, \beta, \gamma, \delta, \varepsilon|=| \alpha+2 \lambda, \beta+2 \mu, \gamma+2 \nu-\mu, \delta+2 \rho-\nu, \varepsilon+2 \sigma \mid$,

$[\alpha, \beta, \gamma, \delta, \varepsilon]\left[\alpha^{\prime}, \beta, \gamma^{\prime}, \delta^{\prime}, \varepsilon^{\prime} \mid\right.$ $=\left[\alpha+\alpha^{\prime}, \beta+\beta^{\prime}, \gamma+\gamma^{\prime}+\beta \alpha^{\prime}, \delta+\delta^{\prime}+\gamma \alpha^{\prime}+\frac{1}{2} \beta \alpha^{\prime}\left(\alpha^{\prime}-1\right), \varepsilon+\varepsilon^{\prime}\right]$.

XXXIII. $(11,12,8,0)(4)$.

$[\alpha, \beta, \gamma, \delta]=\left[\alpha+2 \lambda, \beta+2 \mu_{\gamma} \gamma+2 \nu-\mu, \delta+4 \rho-2 \nu\right]$, $[\alpha, \beta, \gamma, \delta]\left[\alpha^{\prime}, \beta^{\prime}, \gamma^{\prime}, \delta^{\prime}\right\rfloor=\left[\alpha+\alpha^{\prime}, \beta+\beta^{\prime}, \gamma+\gamma^{\prime}+\beta \alpha^{\prime}, \delta+\delta^{\prime}+2 \gamma \alpha^{\prime}+\beta \alpha^{\prime}(\alpha-1)\right]$.

XXXIV. $(19,4,8,0)(4)$.

$[\alpha, \beta, \gamma]=[\alpha+2 \lambda, \beta+8 \mu, \gamma+\varepsilon \nu]$, $\left.[\alpha, \beta, \gamma] \mid \alpha^{\prime}, \beta^{\prime}, \gamma^{\prime}\right]=\left[\alpha+\alpha^{\prime},(-1)^{\alpha^{\prime}} \beta+\beta^{\prime}, \gamma+\gamma^{\prime}\right]$.

XXXV. $(3,20,8,0)(4)$. $[\alpha, \beta, \gamma]=[\alpha+2 \lambda, \beta+8 \mu-4 \lambda, \gamma+2 \nu]$, $[\alpha, \beta, \gamma]\left[\alpha^{\prime}, \beta^{\prime}, \gamma\right]=\left[\alpha+\alpha^{\prime},(-1)^{\alpha^{\prime}} \beta+\beta^{\prime}, \gamma+\gamma^{\prime}\right]$. 


\section{XXXVI. $(3,20,8,0)(4,2)$.}

$$
\begin{gathered}
{[\alpha, \beta, \gamma, \delta]=[\alpha+4 \lambda, \beta+2 \mu, \gamma+2 \nu-\mu, \delta+2 \rho-\nu],} \\
{[\alpha, \beta, \gamma, \delta]\left[\alpha^{\prime}, \beta^{\prime}, \gamma^{\prime}, \delta^{\prime}\right]} \\
=\left[\alpha+\alpha^{\prime}, \beta+\beta^{\prime}, \gamma+\gamma+\beta \alpha^{\prime}, \delta+\delta^{\prime}+\gamma \alpha^{\prime}+\frac{1}{2} \beta \alpha^{\prime}\left(\alpha^{\prime}-1\right)\right] . \\
\quad X X X V I I .(3,4,24,0)(4,2) .
\end{gathered}
$$

$$
\begin{aligned}
& {[\alpha, \beta, \gamma, \delta]=[\alpha+2 \lambda, \beta+2 \mu, \gamma+2 \nu-\mu, \delta+4 \rho-\lambda-2 \nu],} \\
& {[\alpha, \beta, \gamma, \delta]\left[\alpha^{\prime}, \beta^{\prime}, \gamma^{\prime}, \delta^{\prime}\right]=\left[\alpha+\alpha^{\prime}, \beta+\beta^{\prime}, \gamma+\gamma^{\prime}+\beta \alpha^{\prime}, \delta+\delta^{\prime}+2 \gamma \alpha^{\prime}+\beta \alpha^{\prime}\left(\alpha^{\prime}-1\right)\right] .} \\
& \text { XXXVIII. }(11,12,8,0)(4,2) . \\
& {[\alpha, \beta, \gamma, \delta, \varepsilon]=[\alpha+2 \lambda, \beta+2 \mu, \gamma+2 \nu-\mu, \delta+2 \beta-\nu, \varepsilon+2 \sigma-\mu],}
\end{aligned}
$$$$
[\alpha, \beta, \gamma, \delta, \varepsilon]\left[\alpha^{\prime}, \beta^{\prime}, \gamma^{\prime}, \delta^{\prime}, \varepsilon^{\prime}\right]
$$$$
=\left[\alpha+\alpha^{\prime}, \beta+\beta^{\prime}, \gamma+\gamma^{\prime}+\beta \alpha^{\prime}, \delta+\delta^{\prime}+\gamma \alpha^{\prime}+\frac{1}{2} \beta \alpha^{\prime}\left(\alpha^{\prime}-1\right), \varepsilon+\varepsilon^{\prime}\right] \text {. }
$$

XXXIX. $(7,16,8,0)(4,2)$.

$$
\begin{aligned}
& {[\alpha, \beta, \gamma, \delta]=[\alpha+2 \lambda, \beta+2 \mu, \gamma+2 \nu-\mu, \delta+4 \rho-\mu-2 \nu],} \\
& {[\alpha, \beta, \gamma, \delta]\left[\alpha^{\prime}, \beta^{\prime}, \gamma^{\prime}, \delta^{\prime}\right]=\left[\alpha+\alpha^{\prime}, \beta+\beta^{\prime}, \gamma+\gamma^{\prime}+\beta \alpha^{\prime}, \delta+\delta^{\prime}+2 \gamma \alpha+\beta \alpha^{\prime}\left(\alpha^{\prime}-1\right)\right] .} \\
& \text { XL. }(3,20,8,0)(4,2) . \\
& {[\alpha, \beta, \gamma, \delta, \varepsilon]=[\alpha+2 \lambda, \beta+2 \mu, \gamma+2 \nu-\mu, \delta+2 \rho-\lambda-\nu, \varepsilon+2 \varepsilon-\alpha],} \\
& {[\alpha, \beta, \gamma, \delta, \varepsilon]\left[\alpha^{\prime}, \beta^{\prime}, \gamma^{\prime}, \delta^{\prime}, \varepsilon^{\prime}\right]} \\
& =\left[\alpha+\alpha^{\prime}, \beta+\beta^{\prime}, \gamma+\gamma^{\prime}+\beta \alpha^{\prime}, \delta+\delta^{\prime}+\gamma \alpha^{\prime}+\frac{1}{\underline{\alpha}} \beta \alpha^{\prime}\left(\alpha^{\prime}-1\right), \varepsilon+\varepsilon^{\prime}\right] . \\
& \text { XLI. }(3,20,8,0)(4,2) . \\
& {[\alpha, \beta]=[\alpha+4 \lambda, \beta+8 \mu],} \\
& {[\alpha, \beta]\left[\alpha^{\prime}, \beta^{\prime}\right]=\left[\alpha+\alpha^{\prime},(-1)^{\left.\alpha^{\prime} \beta+\beta^{\prime}\right] .}\right.}
\end{aligned}
$$


I dirci Gruppi di grado 32, ciascuno dei quali contiene soltanto 2 eriementi PERMUTABILI CON TUTTI GTI ELEMENTI DEL GRUPPO.

XLII. $(17,2,4,8)(8)$.

$$
\begin{aligned}
& {[\alpha, \beta]=[\alpha+2 \lambda, \beta+16 \mu],} \\
& {[\alpha, \beta]\left[\alpha^{\prime}, \xi^{\prime}\right]=\left[\alpha+\alpha^{\prime},(-1)^{\alpha^{\prime}} \beta+\xi^{\prime}\right] .}
\end{aligned}
$$

XLIII. $(1,18,4,8)(8)$.

$$
\begin{aligned}
& {[\alpha, \beta]=[\alpha+2 \lambda, \beta+16 \mu-8 \lambda,} \\
& {[\alpha, \beta]\left[\alpha^{\prime}, \beta^{\prime} \mid=\left[\alpha+\alpha,(-1)^{\alpha^{\prime}} \beta+\beta^{\prime}\right] .\right.}
\end{aligned}
$$

XLIV. $(9,10,4,8)(8)$.

$[\alpha, \beta, \gamma]=[\alpha+2 \lambda, \beta+2 \mu, \gamma+8 \nu-3 \mu]$,

$[\alpha, \beta, \gamma]\left[\alpha^{\prime}, \beta^{\prime}, \gamma^{\prime}\right]=\left[\alpha+\alpha^{\prime}, \beta+\beta^{\prime},(-1)^{\alpha^{\prime}} \gamma+\gamma^{\prime}+\frac{1}{2}\left|1+(-1)^{\alpha^{\prime}+1}\right| \beta\right]$.

XLV. $(11,20,0,0)(2,2,2)$.

$[\alpha, \beta, \gamma, \delta]=[\alpha+4 \lambda, \beta+2 \mu, \gamma+2 \nu, \delta+2 \rho]$,

$\left.[\alpha, \beta, \gamma, \delta] \mid \alpha^{\prime}, \beta^{\prime}, \gamma^{\prime}, \gamma^{\prime}\right]$

$=\left[\alpha+\alpha^{\prime}, \beta+\beta^{\prime}, \gamma+\gamma^{\prime}+\beta \alpha^{\prime}, \delta+\delta^{\prime}+\gamma \alpha^{\prime}+\frac{1}{2} \beta \alpha^{\prime}\left(\alpha^{\prime}-1\right) \mid\right.$.

XLVI. $(3,12,16,0)(4,2)$.

$[\alpha, \beta, \gamma, \delta]=[\alpha+4 \lambda, \hat{\rho}+2 \mu, \gamma+2 \nu, \delta+2 \rho-\lambda-\mu]$,

$\left[\alpha, \beta, \gamma, \delta \mid\left[\alpha^{\prime}, \beta^{\prime}, \gamma^{\prime}, \delta^{\prime}\right]\right.$

$=\left[\alpha+\alpha^{\prime}, \beta+\beta^{\prime}, \gamma+\gamma^{\prime}+\beta \alpha^{\prime}, \delta+\delta^{\prime}+\gamma \alpha^{\prime}+\frac{1}{2} \beta \alpha^{\prime}\left(\alpha^{\prime}-1\right)\right]$.

XLVII. $(11,4,16,0)(4,2)$.

$[\alpha, \beta, \gamma, \delta]=[\alpha+4 \lambda, \beta+2 \mu, \gamma+2 \nu, \delta+2 \rho-\lambda]$,

$$
[\alpha, \beta, \gamma, \delta]\left[\alpha^{\prime}, \beta^{\prime}, \gamma^{\prime}, \delta^{\prime}\right]
$$$$
=\left[\alpha+\alpha^{\prime}, \beta+\beta^{\prime}, \gamma+\gamma^{\prime}+\beta \alpha^{\prime}, \delta+\delta^{\prime}+\gamma \alpha^{\prime}+\frac{1}{z} \beta \alpha^{\prime}\left(\alpha^{\prime}-1\right)\right] \text {. }
$$

XLVIII. $(15,8,8,0)(4)$.

$[\alpha, \beta, \gamma, \delta, \varepsilon]=[\alpha+2 \lambda, \beta+2 \mu, \gamma+2 \nu, \delta+2 \rho-\mu, \varepsilon+2 \sigma-\rho]$,

$[\alpha, \beta, \gamma, \delta, \varepsilon]\left[\alpha^{\prime}, \beta^{\prime}, \gamma^{\prime}, \delta^{\prime}, \varepsilon^{\prime}\right]$

$$
=\left[\alpha+\alpha^{\prime}, \beta+\beta^{\prime}, \gamma+\gamma^{\prime}, \delta+\delta^{\prime}+\beta \alpha^{\prime}, \varepsilon+\varepsilon^{\prime}+\delta \alpha^{\prime}+\gamma \beta^{\prime}+\frac{1}{2} \beta \alpha^{\prime}\left(\alpha^{\prime}-1\right)\right] \text {. }
$$


XLIX. $(3,20,8,0)(4)$.

$$
\begin{gathered}
{[\alpha, \beta, \gamma, \delta, \varepsilon]=[\alpha+2 \lambda, \beta+2 \mu, \gamma+2 \nu, \delta+2 \rho-\mu, \varepsilon+2 \sigma-\lambda-\beta],} \\
{[\alpha, \beta, \gamma, \delta, \varepsilon]\left[\alpha^{\prime}, \beta^{\prime}, \gamma^{\prime}, \delta^{\prime}, \varepsilon^{\prime}\right]} \\
=\left[\alpha+\alpha^{\prime}, \beta+\beta^{\prime}, \gamma+\gamma^{\prime} \delta+\delta^{\prime}+\beta \alpha^{\prime}, \varepsilon+\varepsilon^{\prime}+\delta \alpha^{\prime}+\gamma \beta^{\prime}+\frac{1}{2} \beta \alpha^{\prime}\left(\alpha^{\prime}-1\right)\right] .
\end{gathered}
$$

L. $(19,12,0,0)(2)$.

$[\alpha, \beta, \gamma, \delta, \varepsilon]=[\alpha+2 \lambda, \beta+2 \mu, \gamma+2 \nu, \delta+2 \rho, \varepsilon+2 \sigma]$,

$[\alpha, \beta, \gamma, \delta, \varepsilon]\left[\alpha^{\prime}, \beta^{\prime}, \gamma^{\prime}, \delta^{\prime}, \varepsilon^{\prime}\right]=\left[\alpha+\alpha^{\prime}, \beta+\beta^{\prime}, \gamma+\gamma^{\prime}, \delta+\delta^{\prime}, \varepsilon+\varepsilon^{\prime}+\delta \alpha^{\prime}+\gamma \beta^{\prime}\right]$.

LI. $(11,20,0,0)(2)$.

$[\alpha, \beta, \gamma, \delta, \varepsilon]=[\alpha+2 \lambda, \beta+2 \mu, \gamma+2 \nu, \delta+2 \rho, \varepsilon+2 \sigma-\mu-\nu]$,

$[\alpha, \beta, \gamma, \delta, \varepsilon]\left[\alpha^{\prime}, \beta^{\prime}, \gamma^{\prime}, \delta^{\prime}, \varepsilon^{\prime}\right]=\left[\alpha+\alpha^{\prime}, \beta+\beta^{\prime}, \gamma+\gamma^{\prime}, \delta+\delta^{\prime}, \varepsilon+\varepsilon^{\prime}+\delta \alpha^{\prime}+\gamma \beta^{\prime}\right]$.

\section{OSSERVAZIONI.}

a) I gruppi IX e $\mathrm{X}$ differiscono in eiò : ognuno dei detti gruppi contiene evidentemente soltanto 4 gruppi ciclici di grado 8 , e questi gruppi ciclici sono divisori normali dentro il gruppo IX e non lo sono dentro il gruppo X.

b) Per assicurarsi che i gruppi XIV e XVIII non sono isomorfi, si pensino i due divisori di grado 8, contenuti rispettivamente nei detti gruppi, e costituiti di tutti gli elementi permutabili con ogni elemento del gruppo principale e si osservi che questi divisori hanno invarianti diversi.

c) Ciascuno dei 19 gruppi che vanno dal numero XXIII al numero XLI possiede un solo divisore Abeliano di grado 16. Gl' invarianti di questi divisori contenuti rispettivamente nei gruppi XXIV e XXVIII sono diversi, e quindi questi gruppi non sono isomorf : la medesima circostanza si presenta per i due gruppi XXV e XXVI e per i due gruppi XXIX e XXXI.

d) Per verificare che i due gruppi XXVII e XXX sono distinti basta osservare che gli elementi del gruppo XXX, che sono fuori del divisore Abeliano di grado 16, in esso gruppo contenuto, hanno tutti lo stesso quadrato che è l'elemento $[0,0,0,0,1]$ e che questo fatto non accade per il gruppo XXVII. 
e) I due divisori di grado 4, contenuti rispettivamente nei due gruppi XXXII e XXXIII, costituiti di tutti gli elementi permutabili con ogni elemento del relativo gruppo principale, non sono isomorfi, e perciò i gruppi principali non sono isomorf.

f) Ciascuno dei tre gruppi XXXVI, XL e XLI contiene soltanto due gruppi ciclici di grado 8, i quali sono divisori normali dentro i gruppi XXXVI e XLI e non lo sono dentro il gruppo XL. Inoltre, per il gruppo XLI accade che ogni suo elemento di ordine 8 è trasformato nel suo inverso da ogni elemento di detto gruppo, che sta fuori del corrispondente divisore Abeliano di grado 16, mentre questo fatto non accade per il gruppo XXXVI.

g) A causa delle inesattezze delle quali ho discorso avanti, nelle tabelle del mio lavoro più volte menzionato non figurano i tre gruppi XXXIV, XXXV e XLI, mentre i due gruppi XLVIII e XLIX vi si trovano scritti due volte. Ecco la natura degli errori ehe io ho commessi. Al principio del n. 38 dell'anzidetto lavoro si legge:

".... e quindi essa (la potenza $E_{4}^{2}$ ) coincide con uno dei quattro elementi :

$$
E_{3}, \quad E_{3} E_{2}, \quad E_{3} E_{1}, \quad E_{3} E_{2} E_{1} .
$$

Io suppongo che si avveri il primo ed il secondo caso, perchè, se fosse altrimenti, assumerei come elemento $E_{4}$ l'elemento $E_{4} E_{3}$ e quindi come elemento $E_{3}$ l'elemento $E_{3} E_{1} . "$

Ma il detto cambiamento, invece di portare i due ultimi casi nei primi due, trasforma i quattro casi ciascuno in sè, e per questa ragione è sfuggita alla mia analisi l'ipotesi, non sempre reducibile, in cui è $E_{4}^{2}=E_{3} E_{1}$, la quale porta precisamente ai tre gruppi XXXIV, XXXV e XLI.

Inoltre, alla fine del n..0 48, dove è detto:

"Dunque, quando è $E_{3}^{\prime}=E_{3}$, si hanno...." bisogna invece dire: "Allora il gruppo di grado 16 generato dagli elementi $E_{5}, E_{4}$, $E_{3}, E_{3}$ non contiene il gruppo $G_{3}=K$, e perciò quando è $E_{3}^{\prime}=E_{3}$ si hanno gruppi che non appartengono alla categoria della quale mi sto occupando."

Questa osservazione era stata da me fatta a proposito del caso generale (pag. 207, lin. 11-12), ma poi ho dimenticato di ripeterla nel caso di $p=2$. 
Io prego quindi il lettore di aggiungere alla tabella II del $\S \mathrm{V}$ (pag. 199) del citato lavoro i tre gruppi XXXIV, XXXV e XLI e di cancellare dalla tabella I del § VI (pag. 216), i due ultimi gruppi che vi figurano: questi due gruppi esistono effettivamente, ma essi coincidono con i due gruppi di grado 32 , che si trovano nella tabella I del § VII (pag. 226).

E con questo restano definitivamente stabiliti tutti i possibili gruppi di grado 32 , i quali risultano in numero di 51 , siccome è stato asserito dal sig. MiLuer (*).

h) Nella tabella della presente Nota i gruppi sono scritti, tenuto il debito conto dell'osservazione precedente, nello stesso ordine nel quale si succedono nelle tabelle del mio citato lavoro. Il lettore può, molto facilmente, trovare i valori degli indici degli elementi che, in ogni singolo caso, corrispondono agli elementi denotati nel detto lavoro con $E_{5}, E_{4}$, $E_{3}, E_{2}, E_{1}$ e stabilire le relazioni poste ivi a fondamento per definire i diversi gruppi.

Palermo, Aprile 1899.

(*) Report on recent Progress in the Theory of the Groups of a finite Order. Bull. of the Amer. Math. Soc., s. 2, v. V, pr. 227-249. 